\title{
INFLUÊNCIA DA RESOLUÇÃO ESPACIAL DE MODELOS DIGITAIS DE TERRENO SOBRE ESTIMATIVAS DE INSOLAÇÃO ANUAL EM UMA ÁREA DE TOPOGRAFIA ACIDENTADA NA SERRA DO CIPÓ (MG)
}

\author{
Julio de Araújo Alves ${ }^{(a)}$, Rafaela Soares Niemann ${ }^{(a)}$, Annia Susin Streher ${ }^{(a, b)}$, Thiago Sanna Freire \\ Silva $a^{(a, b)}$ \\ (a) Universidade Estadual Paulista (Unesp), Instituto de Geociências e Ciências Exatas, Rio Claro, \\ julioaraujoalves@gmail.com; rafaelaniemann@gmail.com; tsfsilva@ rc.unesp.br \\ (b) Universidade Estadual Paulista (Unesp), Instituto de Biociências, Rio Claro; annia.streher@gmail.com
}

\section{Eixo: GEOTECNOLOGIAS E MODELAGEM ESPACIAL EM GEOGRAFIA FÍSICA}

\begin{abstract}
Resumo
A heterogeneidade temporal e espacial da insolação sobre a superfície terrestre determina a dinâmica de uma série de processos ecossistêmicos, como fotossíntese e ciclagem hidrológica. A obtenção de estimativas da insolação anual a partir de modelos digitais de terreno é uma alternativa eficiente ao monitoramento in situ. Atualmente, modelos de elevação com altíssima resolução espacial podem ser gerados a partir de imageamento por veículos aéreos não tripulados, com baixo custo, abrindo novas possibilidades para o estudo dos processos ambientais. $\mathrm{O}$ presente estudo avaliou como o uso de modelos digitais de terreno em diferentes resoluções espaciais pode influenciar a variação espacial das estimativas de insolação anual, em uma região de relevo acidentado. Esta metodologia é importante para se compreender a variação espacial dos processos ecossistêmicos em escala local, em áreas de topografia complexa.
\end{abstract}

Palavras chave: simulação de radiação; veículo aéreo não tripulado, drone, microtopografia, processos ecossistêmicos

\section{Introdução}

A radiação solar tem papel fundamental nas trocas de energia entre a atmosfera e a superfície da Terra (OLIPHANT et al., 2003). A heterogeneidade temporal e espacial da radiação solar incidente sobre a superfície terrestre determina a dinâmica de uma série de processos ecossistêmicos, como a fotossíntese, a ciclagem hidrológica e a temperatura do solo (CHAPIN et al., 2011). Em paisagens topograficamente complexas, com variações significativas na orientação das vertentes, na declividade e na elevação, a distribuição da radiação incidente se relaciona com os padrões do relevo, originando gradientes locais de insolação ao longo do dia e do ano (CHAPIN et al., 2011). Apesar de esforços como a Rede SONDA (www.sonda.ccst.inpe.br) e a Rede de Plataforma de Coleta de Dados (www.crn2.inpe.br/conasat1/redepcd.php), a coleta de dados de radiação solar no Brasil ainda é insuficiente, espacial e temporalmente (PEREIRA et al., 2006). 


\section{OS DESAFIOS DA GEOGRAFIA FÍSICA NA FRONTEIRA DO CONHECIMENTO \\ Instituto de Geociências - Unicamp \\ Campinas - SP \\ 28 de Junho à 02 de Julho de 2017}

A simulação da insolação através de algoritmos computacionais e modelos digitais de terreno tem se mostrado uma alternativa eficiente em termos de custo e tempo (TOVAR-PESCADOR et al., 2006; ARNOLD e REES, 2009). Os VANTs (veículos aéreos não tripulados, comumente chamados de "drones") oferecem a oportunidade de obtenção de dados remotos de altíssima resolução espacial, permitindo embasar estudos dedicados a compreender os processos e mecanismos que ocorrem em escala local na paisagem (CRUZAN et al., 2016).

Desta maneira, o objetivo deste trabalho foi avaliar a influência da resolução espacial dos modelos digitais de terreno gerados a partir de imagens de alta resolução obtidas por VANTs na variação espacial da insolação anual, e entender como a distribuição da radiação pode ser influenciada por variações locais de topografia.

\section{Materiais e métodos}

A área de estudo está localizada na Serra do Cipó, na porção sul da Cadeia do Espinhaço, em Minas Gerais (Figura 1), em uma extensão de 800 por 800 metros entre as latitudes de $19^{\circ} 12^{\prime} \mathrm{S}$ e $19^{\circ} 34^{\prime} \mathrm{S}$ e longitudes de $43^{\circ} 27^{\prime} \mathrm{W}$ e $43^{\circ} 38^{\prime} \mathrm{W}$. A Serra do Cipó está inserida no Escudo Cristalino, e tem variação de altitude entre 800 e 1800 metros (VALENTE, 2009). A paisagem da Serra do Cipó é constituída por rochas predominantemente quartzíticas resistentes a processos de lixiviação e erosão, originando solos rasos e arenosos (IBGE, 2007). A orografia da Cadeia do Espinhaço influencia a formação de chuvas em microescalas, devido às barreiras naturais que auxiliam na ascensão de ar mais úmido para maiores altitudes (SOBREIRO et al., 2015), concentrando boa parte da precipitação anual entre maio e setembro, variando entre 850 e $1.400 \mathrm{~mm}$. A temperatura média anual varia em torno de 18 a $20^{\circ} \mathrm{C}$ no verão e médias abaixo de $15^{\circ} \mathrm{C}$ no inverno. A vegetação da área sobrevoada é dominada pela fisionomia dos campos rupestres, no domínio do Cerrado, geralmente acima dos 900 metros de altitude, com presença de vegetação herbácea e de pequenos arbustos sempre verdes (GIULIETTI et al., 1987). 

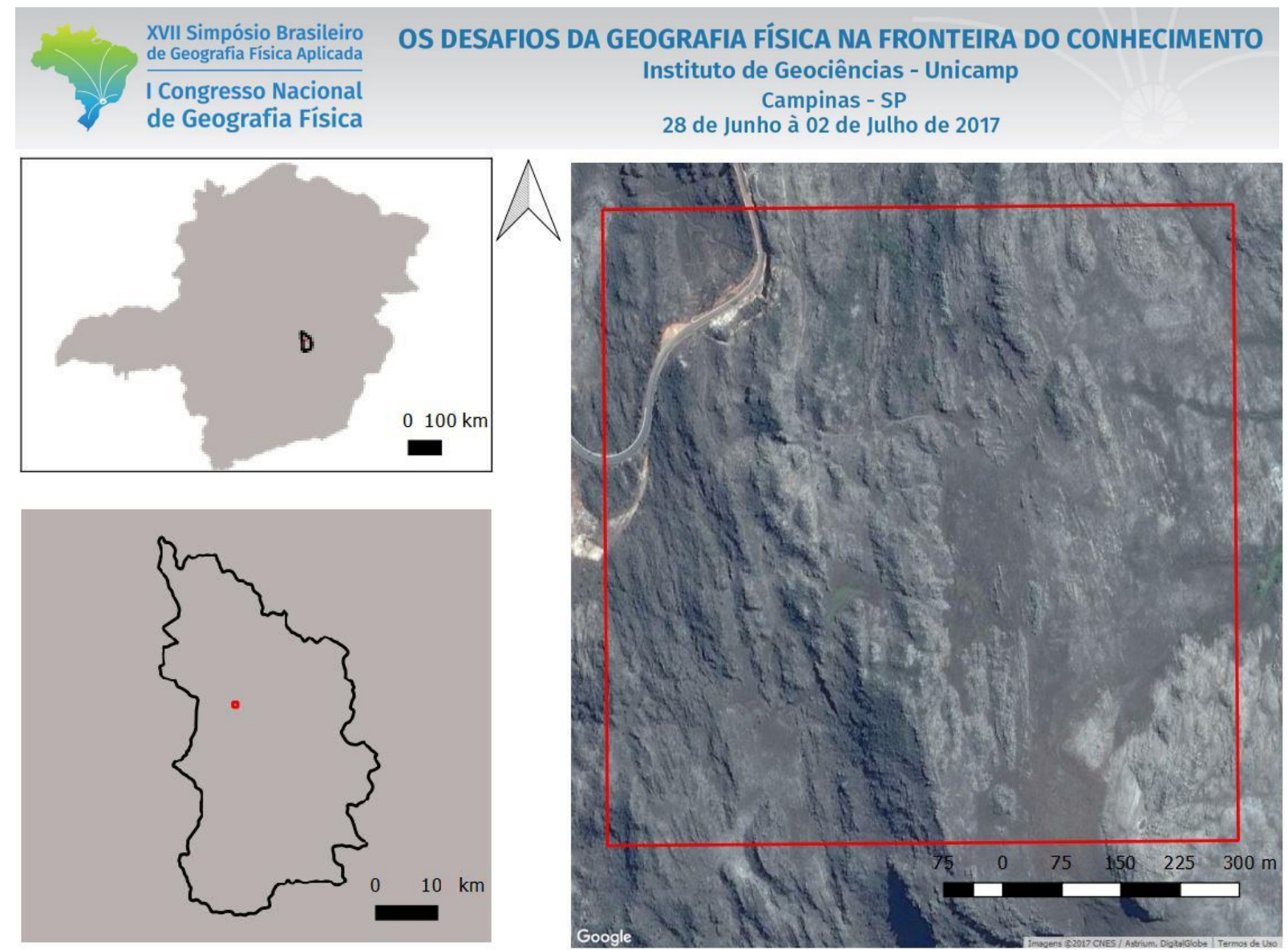

Figura 1: Localização da área de estudo.

Para a realização deste trabalho, foram utilizados Modelos Digitais de Terreno (MDT), gerados a partir de um imageamento adquirido com o auxílio de um Veículo Aéreo Não Tripulado (VANT) de asa fixa, utilizando uma câmera com sensor RGB, que gerou imagens com resolução espacial de $5 \mathrm{~cm}$. Estas imagens foram processadas no software PIX4D para realização de alinhamento e geração de nuvens de pontos tridimensionais através do algoritmo Structure-from-Motion (SfM) (NIEMANN et al., 2016). As nuvens de pontos foram filtradas para a eliminação de objetos de superfície (vegetação, construções, etc.) segundo Niemann et al. (2016), e modelo digital de terreno final foi gerado através de interpolação da superfície em um formato raster utilizando o método spline, regularizado por um método de tensão, realizando uma aproximação espacial baseada nos valores de Z (MITASOVA et al., 2005; NETELER e MITASOVA, 2008). O MDT original foi gerado com resolução espacial de 10 centímetros, e posteriormente agregado para as resoluções de 25 centímetros, 50 centímetros, 1 metro, 5 metros e 30 metros a partir do modelo original, utilizando a função aggregate do "pacote"raster (HIJMANS, 2016), software R. Através desta função, os rasters de resolução mais grossa são gerados através da agregação retangular das células (pixels) menores. A resolução final de 30 metros foi escolhida por equiparar-se aos 
produtos topográficos digitais atualmente disponíveis de forma gratuita para o globo: Shuttle Radar Topography Mission (SRTM, RABUS, 2003) e Aster Global Digital Elevation Model (GDEM, NASA, 2013).

Utilizando cada uma das diferentes resoluções do modelo digital de terreno, calculou-se a insolação anual na superfície, em $\mathrm{kWh} / \mathrm{m}^{2}$, utilizando a ferramenta Area Solar Radiation, encontrada no pacote Spatial Analyst do software ArcGIS 10.3 (www.arcgis.com). Esse método de estimação da insolação não considera a presença de nuvens, mas sim um modelo simplificado de atenuação atmosférica. Os parâmetros utilizados foram baseados nas observações de Rich et al. (1994) e Fu e Rich (1999), e são listados a seguir: (i) "time_configuration" (definição do período utilizado para o cálculo de radiação): 365 dias/ano; (ii) "day_interval" (intervalo de tempo ao longo do ano para o cálculo da posição solar): 1 dia; (iii) "hour_interval" (intervalo de tempo ao longo do dia para o cálculo da posição solar): 0.5 horas; (iv) "slope_aspect_input_type" (indica a fonte de dados para extração dos dados de inclinação e direção das vertentes): FROM_DEM; (v) "diffuse_model_type" (seleção do modelo de radiação difusa): "standard_overcast_sky" (nesse modelo, o fluxo de radiação difusa incidente varia de acordo com o ângulo zenital). Como o objetivo deste foi trabalho foi discutir a variação relativa em função das

diferentes resoluções, e não a precisão absoluta da radiação incidente, os parâmetros "default" da ferramenta foram mantidos, com exceção dos parâmetros "time_configuration" e "day_interval", que foram ajustados para produzir o balanço anual.

\section{Resultados e discussão}

A partir da metodologia apresentada, obtivemos a estimativa da insolação anual a partir de diferentes resoluções espaciais. Foi possível observar que, em resoluções espaciais mais grossas, há uma menor variação espacial da insolação, e quanto mais fina a resolução espacial, mais a estimativa pode variar. A análise dos dados gerados a partir de modelos de alta resolução possibilita a observação de padrões que, em escala regional, dificilmente seriam discriminados (Figura 2). 

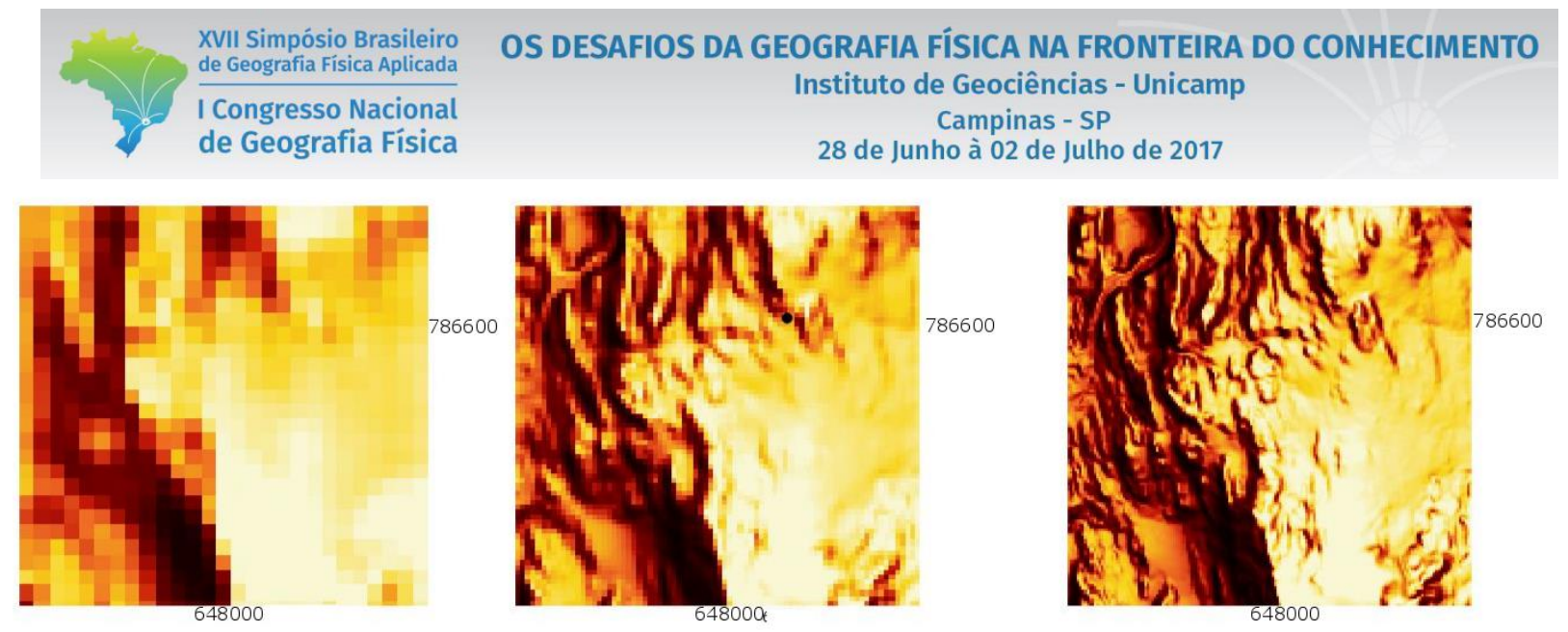

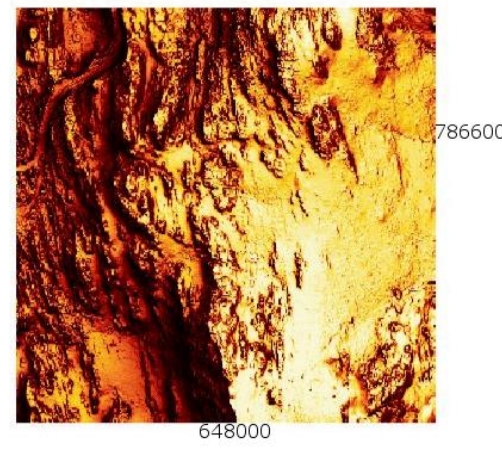

Projeção Univ ersal Transversa de Mercator Datum WGS 84 Zona $23 \mathrm{~S}$
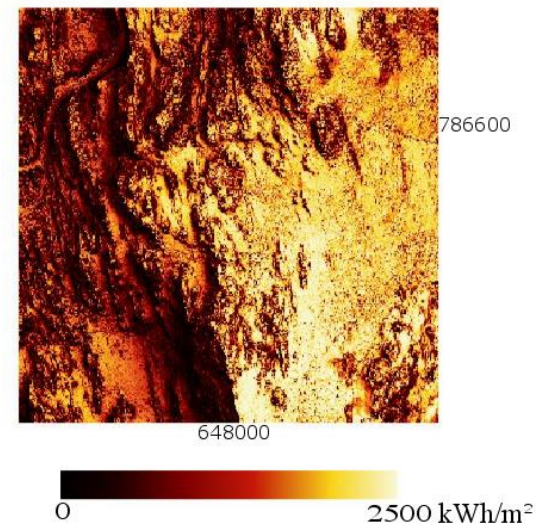

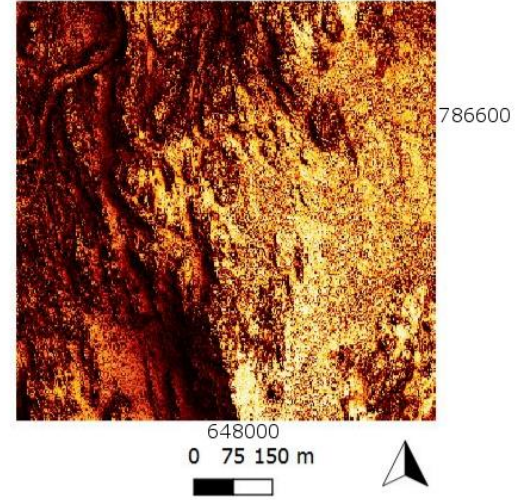

Figura 2: Modelos de estimativa de radiação incidente anual gerados a partir de modelos digitais de terreno de diferentes resoluções espaciais, obtidos através de imageamento por veículo aéreo não-tripulado, mostrando visualmente a variação da estimativa de radiação solar pelo terreno.

Analisando o contexto geral das imagens (Figura 2), é possível observar um padrão de distribuição espacial da radiação incidente ao longo do ano. O padrão encontrado no quadrante sudoeste que se estende a noroeste das imagens está presente desde a resolução mais alta até a mais baixa, indicando que provavelmente essa área receba uma menor quantidade de radiação solar ao longo do ano, por conta do posicionamento geral da vertente em relação a posição do sol. A ocorrência dessa faixa de menor insolação anual é bastante evidente na imagem com resolução espacial de $50 \mathrm{~cm}$, e vai se tornando mais difusa conforme o aumento da resolução. 


$\begin{aligned} & \text { XVII Simpósio Brasileiro } \\ & \text { de Geografia Fisica Aplicada }\end{aligned}$
$\begin{aligned} & \text { I Congresso Nacional } \\ & \text { de Geografia Física }\end{aligned}$
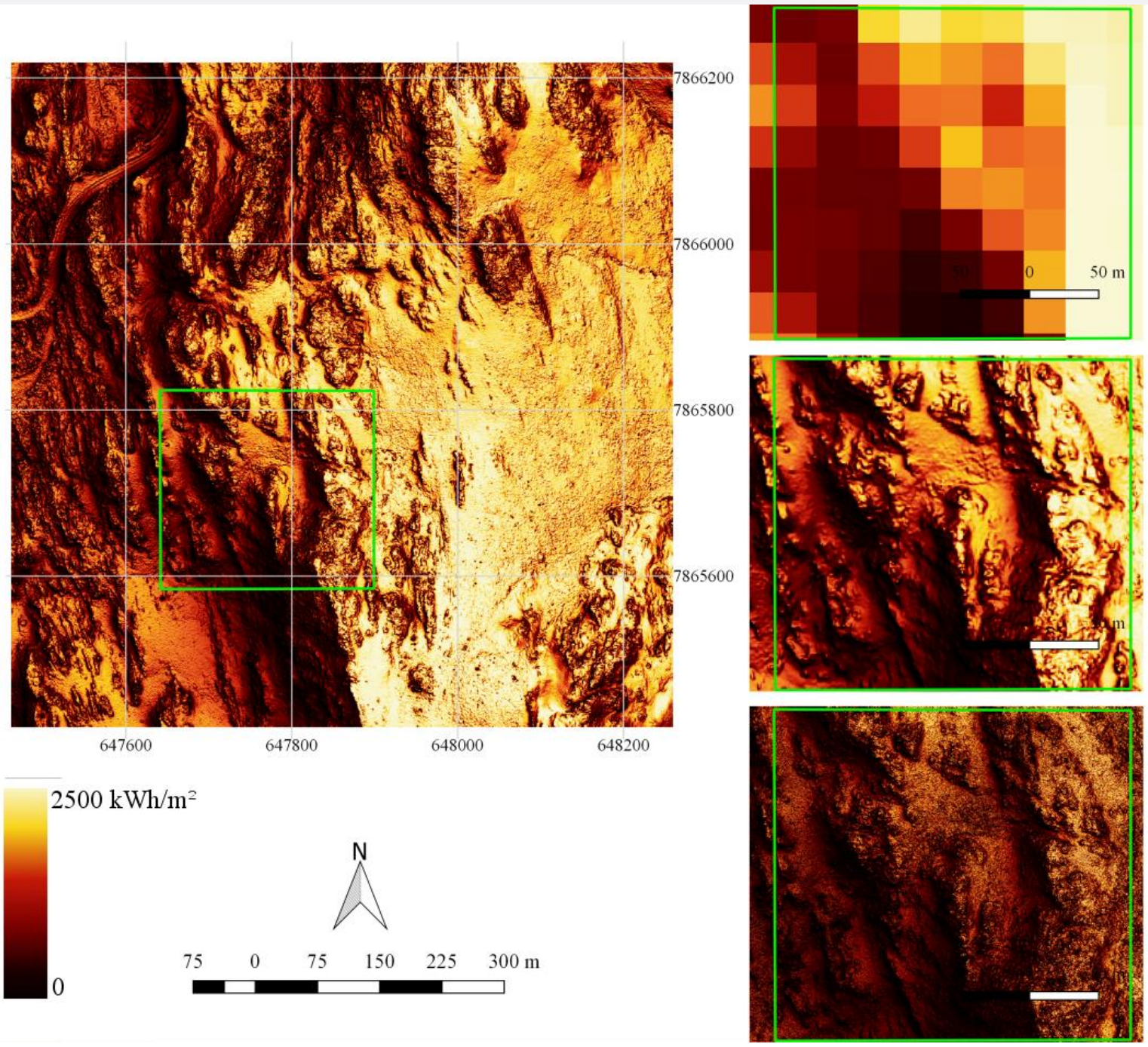

Figura 3: Visão aproximada de uma subárea da região estudada, para as resoluções de $3000 \mathrm{~cm}, 50 \mathrm{~cm} \mathrm{e} 10 \mathrm{~cm}$.

Em termos qualitativos, é possível afirmar que a resolução que permite melhor observação de padrões espaciais de distribuição é a de $50 \mathrm{~cm}$, já que as imagens com resoluções mais finas, como $25 \mathrm{~cm}$ e $10 \mathrm{~cm}$ de resolução, apresentam um volume cada vez maior de ruído que se estende junto aos maiores e menores valores de insolação; enquanto as imagens de 30 m e 10 metros apresentam pouquíssimos detalhes da área em escala local.

Os valores máximos dos pixels da imagem mantêm-se regulares em relação à resolução, enquanto a média dos valores apresenta um aumento uniforme da resolução mais alta para a mais baixa, e o desvio padrão mostra um decréscimo da maior para a menor resolução. O que chama a atenção é o aumento acentuado 
dos valores mínimos, levando a crer que são subestimados na resolução mais alta e/ou superestimados na resolução mais baixa.

Tabela I. Valores máximos e mínimos de insolação anual, em $\mathrm{kWh} / \mathrm{m}^{2}$ extraídos das imagens em diferentes resoluções espaciais.

\begin{tabular}{lrrrrrrr}
\hline & \multicolumn{7}{c}{ Resolução espacial em centímetros } \\
& 10 & 25 & 50 & 100 & 500 & 1000 & 3000 \\
\hline Mínimo & 4 & 40 & 464 & 871 & 1527 & 1800 & 1981 \\
Máximo & 2451 & 2452 & 2451 & 2452 & 2452 & 2451 & 2446 \\
$\begin{array}{l}\text { Média } \\
\text { Desvio }\end{array}$ & 2104 & 2214 & 2256 & 2285 & 2325 & 2341 & 2364 \\
Padrão & 301 & 227 & 194 & 165 & 121 & 103 & 77 \\
\hline
\end{tabular}

Os valores de radiação incidente extraídos dos pixels também compõem o gráfico "boxplot" representado na Figura 3. Ao analisar a variação dos valores de radiação em cada resolução espacial, é possível aferir alguns padrões: a maior variação concentra-se nas imagens de 10 e $25 \mathrm{~cm}$, enquanto a menor variação encontra-se nas imagens de 500, 1000 e $3000 \mathrm{~cm}$. As imagens com 50 e $100 \mathrm{~cm}$ apresentam uma variação similar, assim como seu valor de mediana. 


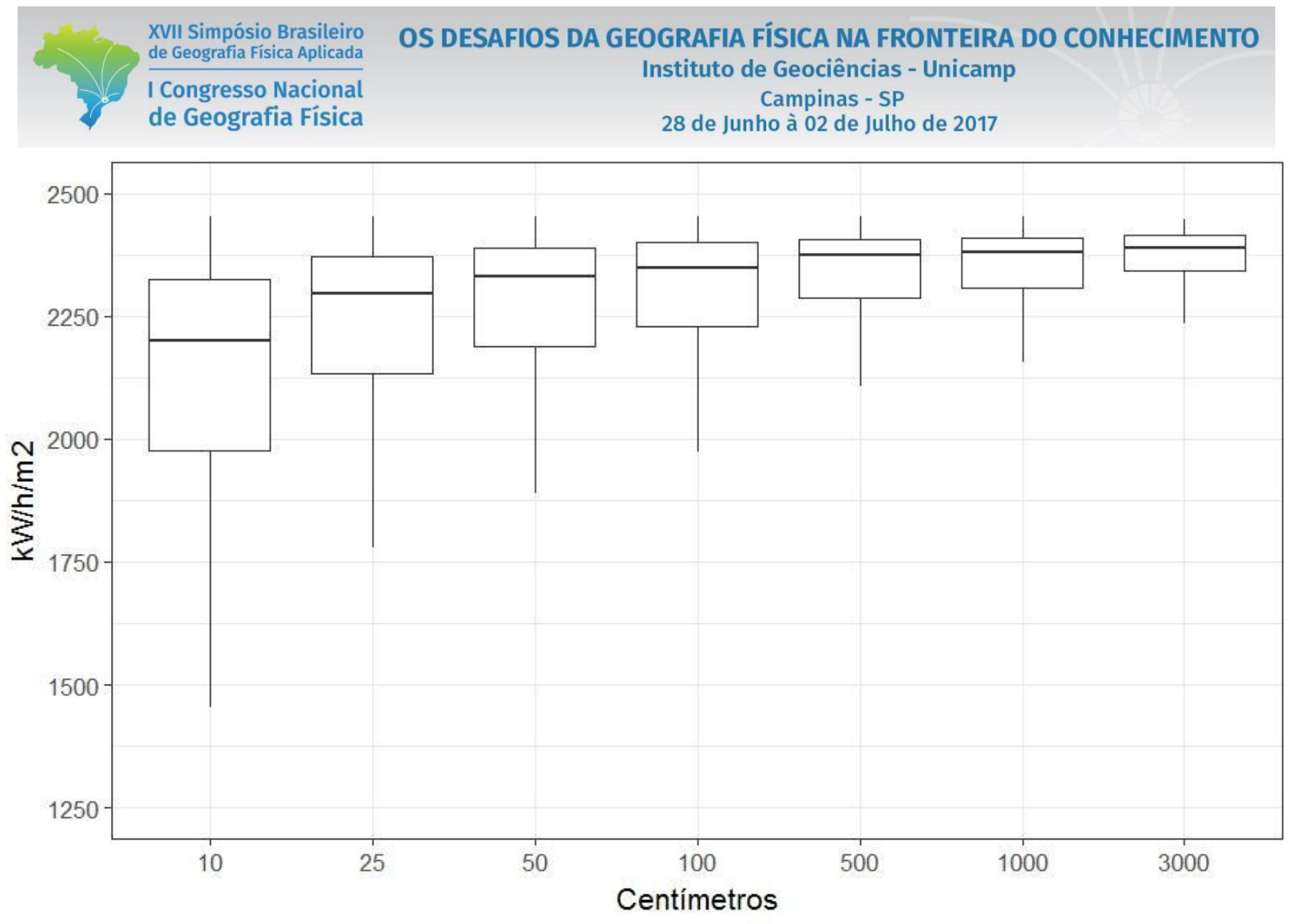

Figura 3: Boxplot das variações entre a radiação incidente por ano em $\mathrm{kWh} / \mathrm{m}^{2}$ e o tamanho do pixel nas diferentes resoluções.

Uma observação que deve ser feita é em relação ao tempo e capacidade de processamento nas diferentes resoluções: as imagens com $10 \mathrm{~cm}$ e $25 \mathrm{~cm}$ de resolução espacial apresentaram uma grande quantidade de ruídos e feições que não representam a realidade local, e também demandaram um altíssimo tempo de processamento (entre 2 e 4 dias em um PC Intel i7 5820k, com 64GB de memória RAM e armazenamento do tipo SSD), tornando-se inviável para estudos na escala do presente trabalho ou mais abrangentes. Assim, os modelos digitais de terreno com resolução entre 50 centímetros e 1 metro mostram o melhor resultado em termos de exigência de processamento versus qualidade dos resultados.

A topografia impõe significativas variações na radiação solar em distâncias curtas. O uso de MDTs permite obter melhores estimativas da radiação solar nestas áreas, levando em consideração os parâmetros topográficos em modelos de transferência de radiação. A simulação fornece boas estimativas da radiação solar, mas é limitada por não considerar a variação na cobertura de nuvens. O uso destas informações pode ser de grande valia para o estudo de processos ecossistêmicos, permitindo uma análise detalhada da diversidade de microhabitats disponíveis para o estabelecimento de espécies vegetais, determinando assim a diversidade e a heterogeneidade em ambientes montanhosos. 


\section{Conclusões}

Neste trabalho, podemos concluir que a metodologia de estimativa da insolação anual através de modelos digitais de terreno de alta resolução pode ser importante para se compreender a variação espacial dos processos ecossistêmicos em escala local, em áreas de topografia complexa. Com dados de resoluções espaciais mais finas, seria possível aferir como estas relações podem influenciar em outros aspectos mais complexos, como processos ecossistêmicos que dependem de microclimas e de feições dos microrrelevos, dificilmente observados em modelos digitais de terreno que possuem dados com resoluções da ordem de dezenas de metros. Os MDTs mais finos, gerados a baixo custo através do uso dos VANTs, auxiliam na compreensão destas áreas e podem colaborar no meio científico, auxiliando na compreensão de novos ecossistemas e detalhes antes não observáveis remotamente em pequena escala. Esta ferramenta nos traz um importante artifício científico, aprimorando os métodos tradicionais de geração de modelos de radiação solar, baseados em medições de estações climatológicas, e realizados através de interpolação, método dependente de uma rede densa de monitoramento para áreas com maior expressividade topográfica, necessitando de um grande volume de dados para uma boa estimativa em topografias complexas.

\section{Agradecimentos}

Esta pesquisa foi financiada pela FAPESP/Microsoft Research (\#2013/50155-0). RS Niemann é bolsista de mestrado CAPES, AS Streher é bolsista de doutorado FAPESP (\#2015/17534-3), e TSF Silva é bolsista de produtividade CNPq (\#310144/2015-9).

\section{Bibliografia}

ARNOLD, N.; REES, G.. Effects of digital elevation model spatial resolution on distributed calculations of solar radiation loading on a High Artic glacier. Journal of Glaciology, v.55, n.194, 2009.

CHAPIN, F.S.; MATSON, P.A.; MOONEY, H.A. Principles of terrestrial ecosystem ecology. New York: Springer, 2002.

CRUZAN, M.B.; WEINSTEIN, B.G.; GRASTY; M.R.; KOHRM, B.F.; HENDRICKSON, E.C.; ARREDONDO, T.M.; THOMPSON, P.G. Small unmanned aerial vehicles (micro-UAVs, drones) in plant ecology. Applications in Plant Sciences, n.4, 2016. 
FU, P.; RICH, P.M. Design and implementation of the Solar Analyst: an ArcView extension for modeling solar radiation at landscape scales. Proceedings of the Nineteenth Annual ESRI User Conference, 1999.

GIULIETTI, A.M.; MENEZES, N.L.; PIRANI, J.R.; WANDERLEY, M.G.L. Flora da Serra do Cipó, Minas Gerais: caracterização e lista das espécies. Boletim de Botânica da Universidade de São Paulo, v.9, 1987.

INSTITUTO BRASILEIRO DE GEOGRAFIA E ESTATÍSTICA. Manual de pedologia. Rio de Janeiro: IBGE, 2007.

MITASOVA, H.; MITAS, L.; HARMON, R. S. Simultaneous spline approximation and topographic analysis for lidar elevation data in open-source GIS. IEEE Geoscience and Remote Sensing Letters, v. 2, n. 4, p. 375-379, 2005.

NASA Jet Propulsion Laboratory (JPL), NASA Shuttle Radar Topography Mission United States 1 arc second. Version 3. 6oS, 69oW. NASA EOSDIS Land Processes DAAC, USGS Earth Resources Observation and Science (EROS) Center, Sioux Falls, South Dakota (https://lpdaac.usgs.gov), 2013.

NETELER, M.; MITASOVA, H. Open Source GIS. Boston, MA: Springer US, 2008. v. 2

NIEMANN, R.S.; SILVA,, T.S.F.; GROHMANN, C.H.; CANCIAN, L.F.; MORELLATO, L.P.C.; LONGHITANO, G.A. Extração de Modelo Digital de Terreno a partir de nuvem de pontos obtida por Veículo Aéreo Não-Tripulado. XI Simpósio Nacional de Geomorfologia - SINAGEO, Maringá 2016.

PEREIRA, E.B.; MARTINS, F.R.; ABREU, S.L.; RÜTHER, R. Atlas brasileiro de energia solar. São José do Campos: INPE, 2006.

RABUS, B.; EINEDER, M.; ROTH, R.; BAMLER, R. The Shuttle Radar Topography Mission-a new class of digital elevation models acquired by spaceborne radar. ISPRS Journal of Photogrammetry \& Remote Sensing, v.57, p.241-262., 2003.

RICH, P.M.; DUBAYAH, R.; HETRICK, W.A.; SAVING, S.C. Using viewshed models to calculate intercepted solar radiation: applications in ecology. American Society for Photogrammetry and Remote Sensing Technical Papers, 1994.

DOI - 10.20396/sbgfa.v1i2017.2221 - ISBN 978-85-85369-16-3 
HIJMANS, R, J,; raster: Geographic Data Analysis and Modeling. R package version 2.5-8. https://CRAN.R-project.org/package=raster, 2016.

SOBREIRO, J.F.F.; SILVA, T.S.F.; STREHER, A.S. Avaliação multi-escala do regime de precipitação da Cadeia do Espinhaço Meridional (Brasil). Congresso de Iniciação Científica da Unesp. Anais..., 2015.

TOVAR-PESCADOR, J.; POZO-VÁZQUEZ, D.; RUIZ-ARIAS, J.A.; BATLLES, J.; LÓPEZ, G.; BOSCH, J.L. On the use of the digital elevation model to estimate the solar radiation in areas of complex topography. Meteorological Applications, n.13, 2006.

VALENTE, E.L. Relações solo-vegetação no Parque Nacional da Serra do Cipó, Espinhaço Meridional, Minas Gerais. Tese (doutorado) - Universidade Federal de Viçosa, MG, 2009. 